Die Kartoffeln waren zu verschiedenen Jahreszeiten angekauft.

Kartoffelwalzmehle zeigten, auf $100 \mathrm{~g}$ Trockensubstanz bezogen, folgende Aschenalkalitäten: $30,11,28,02$ und $30,50 \mathrm{cem}$ N.Säure.

Durch einfache Rechnung kann der Kartoffelgehalt aus den Aschenalkalitätswerten festgestellt werden.

Wie hieraus ersichtlich ist, ist die Differenz der Aschenalkalität zwischen Weizenmehl und Kartoffeln eine beträchtliche und weit größer als die wahre Alkalität der Aschen nach Farnsteiner. Dadurch wird auch der Kartoffelnachweis ein schärferer.

Im hiesigen städtischen und Lebensmittel-Untersuchungsamte ist das Verfahren schon seit 1907 in Gebrauch. Es ist vielleicht überflüssig hervorzuheben, daß meine Versuche sich auf gekochte Kartoffeln beziehen.

Vom theoretischen Standpunkte aus ist ja die Heranziehung der wahren Alkalität nach Farnstein er ganz richtig; für die bei der Kontrolltätigkeit anzuwendenden Methoden ist es aber wichtig, daß sie möglichst einfach und leicht durchführbar sind, da sie nur dann für Massenanalysen in Betracht kommen können.

Ich machte seinerzeit nach der Veröffentlichung der Farnsteiner'schen Bestimmungsmethode auch damit Versuche, fand aber, daß sie etwas umständlicher ist als die allgemein verbreitete Alkalitätsbestimmung; es kommt hinzu, daß die Differenz, wie schon erwähnt, eine geringere ist, als bei der in der gewöhnlichen Weise bestimmten Alkalität, weshalb ich in diesem Falle der gewöhnlichen Methode den Vorzug gebe.

Im ganzen ändert es ja nichts an der Sache, nach welcher Methode man verfährt, der Schwerpunkt liegt eben darin, daß die quantitative Bestimmung der Kartoffel im Brote mit Hilfe der Größe und Differenz der Alkalität der Asche erfolgt; diese Methode ist aber von mir schon im Jahre 1907 veröffentlicht worden.

\title{
Kritische Betrachtungen zum qualitativen und quantitativen Nachweis von Saccharin und über ein neues Verfahren zur qualitativen Bestimmung des Süßstoffes.
}

Von

\section{Klostermann und K. Scholta.}

Mitteilung aus dem Chemischen Untersuchungsamte am Hygienischen Institut der Universität Halle a. S.

[Eingegangen am 17. Dezember 1915.]

\section{Allgemeines.}

Die Identifizierung unbekannter Körper erfolgt auf chemischem Wege durch sogenannte Identitätsreaktionen, die für gerichtliche Feststellungen deshalb so wertvoll sind, weil sie "objektiv" beweisend sind und ohne unser Zutun, ausschließlich auf Grund chemischer Gesetze verlaufen. Diese Reaktionen sind um so beweiskräftiger, je eindeutiger, d. h. je spezifischer für einen bestimmten Stoff sie sind.

Die Natur eines anorganischen Körpers kann man verhältnismäßig leicht und eindeutig durch die Analyse erkennen, für organische Körper ist der Weg nicht so einfach. Bei der grossen Zahl der organischen Verbindungen kann dies nicht Wunder 
nebmen; es kommt noch hinzu, daß sich fast alle von wenigen Grundverbindungen ableiten, in die andere Atomgruppen eingetreten sind und dadurch Körper mit neuen Eigenschaften erzeugt haben. Die Grundverbindungen und die angelagerten Atomgruppen bestehen im Gegensatz zu den anorganischen Verbindungen auch fast immer wieder aus den gleichen Elementen, die nur nach Anordnung und Menge wechseln. Die Elemente sind daher fast immer die gleichen, und da auch die gleichen Atomgruppen häufig wiederkehren, so werden sich naturgemäß auch viele Reaktionen bei den verschiedensten Körpern wiederfinden.

Eine weitere Schwierigkeit ergibt sich aus der Tatsache, daß viele Atomgruppen, sobald sie organisch gebunden sind, ihre Reagierfähigkeit verlieren und mit den sonst typischen Reagentien nicht mehr zu fassen sind. Sie müssen dann erst durch besondere Eingriffe wieder abgespalten werden, bevor man sie nachweisen kann, was nicht immer einfach, aber oft nicht zu umgehen ist.

Die einzelnen Elemente qualitativ nachzuweisen, ist bei der häufigen Wiederkehr der gleichen Elemente meistens zwecklos, und ibre quantitative Bestimmung ist viel zu umständlich, auch genügt hierzu die isolierte Menge des Körpers in den seltensten Fällen.

Berücksichtigt man diese Schwierigkeiten, so wird ein organischer Körper chemisch um so sicherer identifiziert sein, je mehr seiner Atomgruppen nachgewiesen werden, es kommt also darauf an, diese entweder als solche reagieren zu lassen oder sie in andere, gut charakterisierte überzuführen und diese nachzuweisen. Das setzt natürlich eine genaue Kenntnis des Chemismus des betreffenden Körpers und seiner Umwandlungsprodukte voraus, da sonst Fehlschlüsse schwer zu vermeiden sind. Die sogenannten Farbenreaktionen sind daher, soweit ihr Chemismus nicht genau bekannt ist, selten eindeutig und entbehren der nötigen Sicherheit.

Die Schwierigkeit wächst noch, weil bei Nahrungsmitteluntersuchungen oft wur minimale Mengen eines Körpers und noch dazu in unreiner Form vorliegen, sodaß andere Verfahren, z. B. Schmelzpunktbestimmungen, nicht zu Hilfe genommen werden können. Andererseits wird aber die Identifizierung dadurch einfacher, daß bei Nahrungsmitteln nur ein verhältnismäBig kleiner Kreis von Körpern in Frage kommt und eine sehr große Zahl der existierenden von vornherein ausscheidet, weil sie zur Fälschung unverwendbar oder im Handel nicht zu haben sind.

Wir haben uns bemüht, einfache Wege zu suchen, um zu einer eindeutigen Charakterisierung des Saccharins zu gelangen.

\section{$\mathrm{Zum}$ bisherigen $\mathrm{Nachweise} \mathrm{von} \mathrm{Saccharin.}$}

Das Handelsprodukt, mit dem der Nahrungsmittelchemiker fast ausschließlich zu rechnen hat, ist bekanntlich kein einheitliches Produkt, sondern besteht mindestens aus 2 verschiedenen Verbindungen, dem eigentlichen Saccharin oder o-Benzoesäuresulfimid:

und dem p-Benzoesäuresulfamid:<smiles>O=C1NC(=O)C(=O)O1</smiles><smiles>CCCC(=O)C(=O)OO</smiles> 
Außerdem ist noch p-Chlorbenzoesäure gefunden worden, aber sie kommt nicht in allen Präparaten vor, und da sie sich bei den zu besprechenden Reaktionen wie das Amid verhält, so gilt das, was für dieses gesagt wird, auch für jene Säure.

Von diesen Stoffen hat aber nur das o-Benzoesäuresulfimid einen praktischen Wert, nicht das p-Amid, welches $n$ ich $\mathrm{t}$ sü $\mathrm{B}$ schmeckt und deshalb den Bestimmungen des $S$ üfostoffgesetzes rom 7. Juli 1902 nicht unterliegt. Denn nach $\S 1$ sind „Süßstoffe im Sinne dieses Gesetzes alle auf künstlichem Wege gewonnenen Stoffe, welche als Süßmittel dienen können und eine höhere Süßkraft als raffinierter Rohroder Rübenzucker, aber nicht entsprechenden Nährwert besitzen". Da die Paraverbindungen nicht süB schmecken, so fallen sie auch nicht unter die Bestimmungen des Gesetzes, was namentlich bei der quantitativen Frmittelung von Saccharin bislang nicht berücksichtigt worden ist. Auch qualitativ hat man sich gewöhnlich mit dem Nachweise der Sulfonsäure begnügt. Will man aber dem Gesetze genau Rechnung tragen, so muß vor Ausführung der Bestimmung oder Reaktion die Paraverbindung ausgeschaltet werden, um einen eindeutigen Saccharinnachweis zu gewährleisten.

Zum Nachweise in Nahrungsmitteln muß das Saccharin zunächst isoliert werden, dies geschieht allgemein durch Ausschütteln der sauren wässerigen Lösung mit einer Mischung von Äther-Petroläther. Nach dem Verdunsten dieses Lösungsmittels bleibt ein Rückstand, der niemals rein, sondern mehr oder weniger verunreinigt ist durch Stoffe, die je nach dem Ausgangsmaterial sehr verschieden sein können. Zu nennen wären bauptsächlich Peptone, Milchsäure, Gerbstoffe, ätherische Öle, Maltol, Fettsäuren, Harze, Bitterstoffe, Weinsäure, Citronensäure, Äpfelsäure, Bernsteinsäure, Zucker, Salicylsäure, Benzoesäure, Formalin usw.

Es sind deshalb auch verschiedene Reinigungsverfahren vorgeschlagen worden, z. B. mit Kaliumpermanganat oder Bleiessig oder bei Anwesenheit flüchtiger Stoffe die Destillation mit Wasserdampf. Es sind dies aber zeitraubende Arbeiter, die zudem nicht immer zum Ziele führen.

Die erste Prüfung auf Saccharin erfolgt wohl stets durch den Geschmack. Dieser ist natürlich rein subjektiv und das Ergebnis nicht eindeutig, da der süße Geschmack durch ätherische Öle, Gerbstoffe, Maltol, Salicylsäure und namentlich Bitterstoffe, z. B. bei stark gehopften Bieren, verdeckt werden kann. Aber auch wenn dies nicht der Fall ist, genügt das subjektive Empfinden allein nicht, um die Gegenwart von Saccharin als erwiesen anzusehen. Jedenfalls darf sich der Chemiker hiermit nicht begnügen, wenn ein positives Ergebnis weitere Folgen nach sich ziehen kann.

Es sollen daher zunächst die gebräuchlicben Reaktionen, die bisher angewandt wurden, besprochen werden.

Eine bäufig angestellte Prüfung beruht auf dem Nachweise der Sulfogruppe durch Schmelzen des Rückstandes mit Soda und Salpeter und Fällung der Schwefelsäure mit Chlorbarium. Nach diesem Verfahren wird aber nicht nur jede $\mathrm{Sulfo-}$ gruppe, sondern allgemein jede schwefelhaltige Substanz nachgewiesen, da alle Schwefelverbindungen hierbei Schwefelsäure liefern. Ferner geben die im Saccharin enthaltenen Paraverbindungen, die für die Beurteilung an sich gleichgültig sind, ebenfalls Schwefelsäurereaktion, und der positive Ausfall der Reaktion ist daher nicht beweisend dafür, daß eine Orthosulfosäure wie im Imid vorliegt. Das gleiche gilt natürlich auch für die quantitative Bestimmung. 
Außerdem fand Vollhase ${ }^{1}$, daß bei geringen Mengen Saccharin die Reaktion versagt, weil überschüssige Salpetersäure oder Kaliumnitrat das Ausfallen des Bariumsulfats verhindern. Er empfiehlt daher die Verwendung von Kaliumchlorat an Stelle von Salpeter. Ferner stellte er fest, daß bei Verwendung von Glas- oder Porzellangefäßen die Wandungen Spuren von Schwefelsäure abgeben, was zu Irrtümern führen kann.

Der gleiche Fehler des Nachweises fremden Schwefels haftet auch jenen Methoden an, welche den Schwefel der Sulfogruppe auf andere Weise nachzuweisen versuchen. Das sind die v. Maler'sche Reaktion ${ }^{2}$, nach der das Saccharin mit metallischem Natrium oder Kalium erwärmt und das Sulfid mit Nitroprussidnatrium nachgewiesen wird, und die Methode nach G. Possetto und G. Isoglio ${ }^{3}$ ), die den Schwefel als Schwefelwasserstoff mit Hilfe der Methylenblaureaktion sichtbar macht.

Die gebräuchlichste Reaktion ist wohl die Überführung des Saccharins in Salieylsä ure durch Schmelzen mit Natriumhydroxyd bei $210-215^{0}$ nach F. Wirthle ${ }^{4}$ ). Sie ist schon wesentlich eindeutiger als der Nachweis der Sulfogruppe, da sich Salicylsäure nur aus der Orthostellung bildet. Aus den Paraverbindungen bilden sich zwar analoge Verbindungen - aus dem Parasulfobenzamid also Paraoxybenzoesäure -, aber sie geben mit Eisenchlorid nicht die bekannte Violettfärbung, sondern nur Gelbfärbung oder gelben amorphen Niederschlag und sind daher mit der Orthosäure nicht zu verwechseln.

Das Schmelzen mit Natriumbydroxyd hat die Wirkung, daß die Imidgruppe abgespalten und die Sulfogruppe dureh die OH-Gruppe ersetzt wird. 'Der Verlauf der Reaktion ist fast quantitativ, und da die Reaktion mit Eisenchlorid sehr empfindlich ist, so gelingt der Nachweis selbst geringer Saccharinmengen gut.

Das Verfahren hat aber den Nachteil, daß es bei Gegenwart von Salicylsäure, die oft als Konservierungsmittel in Nahrungsmitteln vorkommt, unmittelbar nicht anwendbar ist. In solchen Fällen muß der Rückstand von der Äther-Petrolätherausschüttelung, der Salicylsäure neben Saccharin enthält, zunächst mit Kaliumpermanganat und Schwefelsäure behandelt werden, um die Salicylsäure zu zerstören, wobei man gleichzeitig erreicht, daß auch viele der übrigen Verunreinigungen entfernt werden.

Es ist ferner zu beachten, daß das Eisenchlorid ein Gruppenreagens für fast sämtliche Phenole, Phenolhomologe und viele Phenolderivate ist, wobei verschiedene Farben, wie blau, violett oder rot und vielfach auch Übergangsfarben auftreten, die violette und ähnliche Töne umfassen. Solche Verbindungen können a priori in den Ätherauszug gelangt sein, wie z. B. das von $\mathrm{Brand}^{5}$ ) entdeckte Maltol, das sich in Brot und Cerealien findet, oder das von $\mathrm{Backe}^{6}$ ) aufgefundene Isomaltol, das durch Karamelisation aus Zucker oder Stärke entsteht. Sie können aber auch später bei der Alkalischmelze aus den verschiedensten Verbindungen entstehen, namentlich wenn die Temperatur zu sehr gesteigert wird. Die Reaktion ist daher nicht immer eindeutig,

1) Chem,-Ztg. 1913, 37, 425 . 10, 499 .

2) Chem.-Centralbl. 1905, I, 564; Chem.Ztg. Repert. 1904, 28, 270; Diese Zeitschr. 1905,

3) Diese Zeitschr. 1913, 26, 60.

4) Chem.Ztg. 1901, 25, 316 .

$\left.{ }^{5}\right)$ Ber. D. Chem. Ges. 1894, 27, 806.

6) Chem.-Ztg. 1910, 34, 289. 
und Vollhase ${ }^{1}$ empfahl deshalb an Stelle der Eisenchloridreaktion die Reaktion nach Jorrissen ${ }^{2}$ ). Danach wird der Ätherrückstand mit einem Tropfen 15\%-iger Natronlauge und 2-3 ecm Wasser aufgenommen und in ein Reagensglas filtriert. Dann setzt man 5 Tropfen 50\%-iger Essigsäure, 5 Tropfen 10\%-iger Kaliumnitritlösung und 1 Tropfen 1\%.iger Kupfersulfatlösung hinzu und erhitzt auf dem siedenden Wasserbade. Nach 8 Minuten tritt Rotfärbung ein. F. Schott ${ }^{3}$ ) prüfte diese Reaktion und fand, daß freie Mineralsäuren und verschiedene organische Säuren wie Weinsäure und Citronensäure die Reaktion verhindern.

Abgesehen von diesen Unsicherheiten ist die Überführbarkeit in Salicylsäure auch nur beweisend dafür, daß eine in Orthostellung substituierte Benzoesäure vorliegt.

Das Verfahren hat aber den Nachteil, daß es umständlich ist und daß zum eindeutigen Nachweise des Saccharins auferdem noch die Sulfongruppe und Imidgruppe nachzuweisen ist.

Wir haben uns deshalb bemüht, hierfür einfachere Reaktionen zu ermitteln, die auch durch die angeführten Stoffe nicht beeinflußt werden.

Zunächst sollen noch zwei Reaktionen besprochen werden, die aber seltener angewendet werden; es sind die Reaktionen von Börnstein ${ }^{4}$ ) und von Kastle ${ }^{5}$ ).

Nach Börnstein soll eine kleine Menge Saccharin mit einem geringen Überschuf von Resorcin und einigen Tropfen Schwefelsäure erhitzt werden. Die Mischung färbt sich gelb, rot, dann dunkelgrün und gerät unter reicblicher Entwickelung von schwefliger Säure in starkes Wallen. Das ist 2-3-mal zu wiederholen. Nach dem Abkühlen soll mit Wasser verdünnt werden, wobei eine gelblich gefärbte Lösung entsteht, die nach dem Alkalisieren starke Fluoreszenz von grün zu rotgelb zeigt.

Der Körper, welcher hierbei entsteht, ist Sulfofluorescein:<smiles>OCCCCCCCCc1ccccc1O</smiles>

Er ist also wie Fluorescein zusammengesetzt und zeigt auch dieselbe Art von Fluoreszenz, die aber bei der Sulfoverbindung noch stärker ausgeprägt ist. Die Reaktion ist bei Verwendung von reinem Saccharin sehr empfindlich und noch bei $1 \mathrm{mg}$ Saccharin deutlich zu erkennen.

Die Darstellung des Farbstoffes gelang Remsen ${ }^{6}$ ) in etwas größeren Mengen auf folgende Weise: Das Imid wurde zunächst durch Kochen mit Salzsäure in das saure Ammoniumsalz der o-Sulfobenzoesäure übergefübrt. Dieses wurde mit Resorcin auf $180^{\circ}$ erhitzt, wobei das Ammoniumsalz der Dioxybenzoylbenzensulfosäure von der Formel $(\mathrm{OH})_{2} \cdot \mathrm{C}_{6} \mathrm{H}_{3} \mathrm{CO} \cdot \mathrm{C}_{6} \mathrm{H}_{4} \cdot \mathrm{SO}_{2} \mathrm{OH}$ entstand. Aus diesem wurde durch Kochen mit Bariumcarbonat das Bariumsalz und durch Zusatz von Schwefelsäure die freie Di-

1) Chem.Ztg. 1913, 37, 426.

$\left.{ }^{2}\right)$ Bull. Acad. Roy. Belg. 1882, 3, 259.

3) Diese Zeitschr. 1911, 22, 728.

4) Zeitschr. f. analyt. Chem. 1890, 27, 165.

5) Chem. Zentralbl. 1906, I, 1575.

6) Amer. chem. Journ. 9, 372 und 11, 73 u. 332. 
oxybenzoylbenzensulfosäure gewonnen. Beim Erhitzen auf $180^{\circ}$ entstand aus dieser unter Austritt von Wasser und unter Abspaltung eines Moleküls o-Sulfobenzoesäure das Sulfofluorescein.

Die Spaltung des Imids in das saure Ammoniumsalz der o-Sulfobenzoesäure, die Bildung des Ammoniumsalzes der Dioxybenzoylbenzensulfosäure, die weitere Bildung: der freien Säure, die Wasserabspaltung und der Übergang in den Farbstoff, diese ganze Reihenfolge wird sich auch bei der Börnstein'schen Reaktion abspielen. Eine Spaltung des Imids wird dureh die Schwefelsäure jedenfalls bewirkt, und für diesen Vorgang muß schon Säure vorhanden sein. Sie wird auch bei der zur Farbstoffbildung notwendigen Wasserentziehung mitwirken, aber dies gelingt auch auf anderem Wege ohne Schwefelsäure. Diese ist nämlich für die Bildung der Dioxybenzoylbenzensulfosäure aus 0 -Sulfobenzoesäure und Resorcin nicht günstig, denn Charles Blackshear ${ }^{1}$ ) erhielt beim Erhitzen von Dioxybenzoylbenzensulfosäure mit konc. Schwefelsäure nur sehr wenig Farbstoff, wäbrend der größte Teil in o-Sulfobenzoesäure zurückverwandelt wurde. Nebenher bildete sich eine dunkelbraune unlösliche Masse. Ähnliche Zersetzungsprodukte bilden sich auch bei der Börnsteinschen Reaktion und machen sie oft unsicher, was als ein Nachteil dieser Reaktion anzusehen ist. Da Börnstein für seine Reaktion sehr hohe Temperaturen vorschreibt, so tritt leicht Verkohlung ein. Schon reines Saccharin bräunt sich bei Anstellung der Reaktion, sind aber größere Mengen anderer organiseher Substanzen vorhanden, z. B. ätherische Öle, so erbält man oft schwarze Massen, welche eine Wahrnehmung der Fluoreszenz so gut wie unmöglich machen.

Ein weiterer Nachteil der Börnstein'schen Reaktion ist der, daß auch verschiedene andere Stoffe mit Resorcin und Schwefelsäure erbitzt in alkalischer Lösung die grüne Fluoreszenz geben. So fand $H o k e r^{2}$ ) sogar, daß Resorcin allein, mit Schwefelsäure erhitzt, wenn mehrere Centigramme angewendet werden, auch fluoreszierende Verbindungen liefert. Nach Börnstein ${ }^{3}$ ) kann man das vermeiden, wenn man nur 1 Centigramm Resorcin für die Reaktion verwendet. Es gibt aber noch eine große Anzahl Stoffe, die gleichfalls Fluoreszenz zeigen.

Haas ) versuchte die Börnstein'sche Reaktion zum Nachweise von Saccharin in Süßweinen zu verwenden. Er fand, daß Äpfelsäure, Bernsteinsäure, Zitronensäure, Saccharose ebenfalls grüne Fluoreszenz geben.

Ganther ${ }^{5}$ ) untersuchte 12 Biere auf Saccharin, bekam bei allen einen positiven Ausfall der Reaktion und fand, daß Hopfenharz die Fluoreszenz gibt.

Commanducci ${ }^{6}$ ) ermittelte, dah auch Glykose und Saccharose Fluoreszenz geben.

Wir untersuchten eine Reihe von Stoffen (Tab. S. 75) und erhielten Fluoreszenz bei Verwendung von Benzaldehyd, Benzoesäure, Naphthol, Hexamethylentetramin, Vanillin, Cumarin, Pepton, Zuckerfarbe, Zimtöl, Nelkenöl, Senföl, Paprikaöl, Anisöl, Catechu, Kampfer, Hopfenextrakt. Daraus ergibt sich ohne weiteres, daß die Re-

1) Amer. ehem. Journ. 14, 455.

2) Ber. D. Chem. Ges. 1888, 21, 3395.

3) Zeitschr. f. analyt. Chem. 1889, 28, 352.

$\left.{ }^{4}\right)$ Zeitschr. f. analyt. Chem. 1889, 28, 713 .

5) Zeitschr. f. analyt. Chem. 1893, 32, 309.

•) Chem. Zentralbl. 1910, II, 1951. 
aktion nicht eindentig ist und außer mit Saccharin auch mit vielen anderen Stoffen eintritt, die in Nahrungsmitteln vorkommen und in den Ätherauszug gelangen. Das Eintreten der Reaktion durch Körper der verschiedensten Zusammensetzung ist sicher auf tiefer greifende Zersetzungen durch die konc. Schwefelsäure zurückzuführen.

Daß auch Phthalsäure und Phthalimid die gleiche Reaktion geben, ist selbstverständlich, aber sie kommen in Nahrungsmitteln nicht vor und sind deshalb ohne Bedeutung.

Kastle $\left.{ }^{1}\right)$ modifiziert die Börnstein'sche Reaktion in der Weise, daß er statt Resorein Pbenol anwendet. Dabei entsteht Sulfophenolphthalein:<smiles>CCCC(CC)(CCO)OOS(=O)(=O)O</smiles>

welches in saurer Lösung gelbrot, in alkalischer dem Phenolphthalein ähnlich blaurot gefärbt ist. Von diesem unterscheidet es sich chemisch auch nur dadurch, daf die Carboxylgruppe, welche das innere Anbydrid bildet, durch die Sulfogruppe ersetzt ist.

Diese Reaktion hat den Vorteil, daß sie bei niedrigerer Temperatur vor sich geht. Kastle schreibt $170-180^{\circ}$ vor. Es gibt aber außer dem Saccharin noch verschiedene Stoffe, welche eine Rotfärbung geben, die nicht von Sulfophthalein herrührt. Kastle erhielt positive Reaktion nur bei Saccharin und Vanillin; Phthalsäure und Phthalimid geben sie natürlich auch, können aber aus dem oben angegebenen Grunde bei Nahrungsmitteln vernachlässigt werden. Wir erhielten Rotfärbung mit Benzaldehyd, Vanillin, Nelkenöl, Zimtöl, Zimtsäure, Benzoeharz, Kolophonium, Tannin (vergl. Tabelle S. 75). Als sicher kann die Reaktion deshalb nicht angesehen werden.

Auch bei dieser Reaktion treten oft braune Massen auf, doch wird die blaurote Farbe dadurch nicht so leicht verdeckt wie die Fluoreszenz.

Betrachten wir den theoretischen Chemismus der Reaktionen nach Börnstein und nach $\mathrm{Kastle}$, so kann man sagen, daß sie bis zu einem gewissen Grade eindeutig sind, aber die vorgeschriebene Ausführung nicht praktisch ist, was in erster Linie auf die Anwendung von Schwefelsäure und Erhitzung auf zu hohe Temperaturen zurückzufübren ist, wodurch tiefergehende Zersetzungen mancher Körper nicht zu umgehen sind. Die Ausschaltung der Schwefelsäure ist auch deshalb wünschenswert, weil die Kondensation bei ihrer Gegenwart nur sehr träge verläuft. Uns war es nicht möglich, selbst bei Anwendung größerer Saccharinmengen und verschiedener Temperaturen Sulfofluorescein und Sulfophenolphtbalein $\mathrm{zu}$ isolieren. Sie können also nur in ganz geringer Menge entstanden sein, und Blackshear ${ }^{2}$ ) und Sohon ${ }^{3}$ ) fanden ebenfalls, daß faßbare Mengen der Farbstoffe auf diesem Wege nicht gebildet werden.

1) Chem. Zentralbl. 1906, I, 1575.

$\left.{ }^{2}\right)$ Amer. chem. Journ. 14, 455.

$\left.{ }^{3}\right)$ Amer. chem. Journ. 20, 257. 


\section{Neue Reaktion zum Nachweise von Saccharin.}

Es hatte sich also als notwendig erwiesen, die Schwefelsäure auszuschalten, das Anhydrid auf andere Weise herzustellen und dieses mit Phenol zu kondensieren. Diesen Weg suchten wir zu finden.

Da weder das Saccharin noch die o-Sulfobenzoesäure sich mit Phenol, auch nicht beim Erhitzen, kondensieren lassen, dies aber mit der o-Sulfobenzoesäure in Form ihres Anhydrids gelingt, so mußte das Saccharin zunächst in o-Sulfobenzoesäure und diese in das Anhydrid übergeführt werden. Das erste gelingt leicht durch Behandeln mit Salzsäure, wobei das saure Ammoniumsalz der Säure entsteht.

Aus diesem stellte Sobon ${ }^{1}$ ) das Anhydrid dar, indem er es mit Phosphorpentachlorid erhitzte und das Reaktionsgemisch nach Behandeln mit Wasser mit Benzol extrahierte. Er fand, daß das Anhydrid bei 129,5 sublimierte. Diese Arbeitsweise läßt sich aber für unsere Zwecke nicht gebrauchen, da sie zu umständlich und zeitraubend und mit den zu Gebote stehenden kleinen Mengen nicht ausführbar ist.

Bei unseren Versuchen fanden wir, dab sich das saure Ammoniumsalz der o-Sulfobenzoesäure schon durch Behandeln mit Phosphorsäureanhydrid in das Anhydrid verwandeln läßt und zwar ohne Erhitzen, da die Reaktionswärme genügt. Ist Phenol zugegen, so gelingt auch gleichzeitig. die Kondensation mit Phenol, wodurch sich die Reaktion sehr einfach gestaltet. Es genügt nämlich, das saure Ammoniumsalz der o-Sulfobenzoesäure in Phenol zu lösen und diese Lösung mit Phosphorsäureanhydrid zu behandeln, wobei ohne weiteres Sulfophenolphthalein entsteht.

Die Reaktion führt man so aus, daß man etwas Saccharin mit Salzsäure von etwa $10 \%$ einige Minuten kocht. Die Lösung wird in eine Krystallisierschale gebracht und zur Trockne verdampft. Der Rückstand wird in wenig flüssiger Karbolsäure $(90 \%)$ gelöst und die Lösung auf Phosphorsäureanbydrid getropft, von dem man eine Messerspitze voll in einen kleinen Porzellantiegel getan hat. Es erfolgt unter Erwärmen eine beftige Reaktion, wobei sich ein dunkelrot gefärbtes Produkt bildet. Wird dieses in Wasser gelöst, so erhält man eine gelbliche Lösung, die auf Zusatz von Alkali die bekannte blaurote Färbung der Phthaleine zeigt.

Um die Empfindlichkeit der Reaktion zu prüfen, wurde eine bestimmte Menge Saccharin durch Kochen mit Salzsäure in das saure Ammoniumsalz umgewandelt und dieses in Phenol gelöst; durch Verdünnen mit Phenol wurden Lösungen von verschiedener Konzentration hergestellt. Die Prüfung ergab, dab $1 \mathrm{mg}$ Saceharin noch eine sehr deutliche Färbung gibt, also sicher zu erkennen ist.

Um die Brauchbarkeit der Reaktion bei Nahrungsmitteluntersuchungen festzustellen, mußte zunächst ermittelt werden, ob auch andere Stoffe als Saccharin den gleichen oder einen ähnlichen Farbstoff liefern können. Es wurde daher eine große Zahl von Stoffen auf ihr Verhalten bei der Reaktion geprüft. Die Ergebnisse sind in der folgenden Tabelle zusammengestellt.

1) Amer* chem. Journ. 20, 257. 


\begin{tabular}{|c|c|c|c|c|c|c|c|c|}
\hline & & & & & & $\begin{array}{l}\text { Reaktion nach } \\
\text { Börnstoin }\end{array}$ & $\begin{array}{l}\text { Reaktion nach } \\
\text { Kastle }\end{array}$ & $\begin{array}{c}\text { Unsere } \\
\text { Reaktion }\end{array}$ \\
\hline Macis & . & . & , & . & . . & - & - & - \\
\hline Nelkenöl . & . & . & . & . & . . & deutlich & schwach rot & - \\
\hline Kümmelöl . . & . & . & . & . & . . & - & - & - \\
\hline Zitronenöl. . & . & . & . & . & . . & - & - & - \\
\hline Anisöl . . & . . & . & . & . & . . & sehr stark & - & - \\
\hline Zimtöl. . . & . . & . & . & . & . . & stark & stark & - \\
\hline Wacholderöl & . . & . & . & . & . . & - & - & - \\
\hline Senfol . . & . & . & . & . & . . & dentlich & - & - \\
\hline Paprikaöl . . & - . & . & . & . & . . & sehwach & - & - \\
\hline Perubalsam . & . & . & . & . & . . & - & - & - \\
\hline Salicin . . & . & . & . & . & . . & - & - & - \\
\hline Campher. & . & 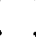 & . & . &. . & schwach & - & - \\
\hline Cumarin . . & . & . & . & . & . . & sebwach & - & - \\
\hline Salicylaldehyd & . & $\because$ & . & . & . . & deutlich & dentlich & - \\
\hline Catechuharz . & . . & . & . & . & . . & deutlich & - & - \\
\hline Benzoeharz . & . . & . & . & . & .. . & - & schwach rot & - \\
\hline Zimtsäure . & . . & . & . & . & . . & - & dentlich & - \\
\hline Urotropin . &. & . & . & . & . . & deutlich & - & - \\
\hline Benzoesäure . & . . & . & , & . & . . & deutlich & - & - \\
\hline Salicylsäure . & . . & 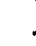 & . & . & .. & - & - & $一$ \\
\hline Lupulinauszug & . & . & . & . & . & deutlich & - & - \\
\hline Styrax. . & . . & . & . & . & . . & - & - & - \\
\hline Pepton. . & . . & . . & . & . & . . & deutlich & - & - \\
\hline Hopfenöl . & . . & . & . & . & . . & - & - & - \\
\hline Saponin . . &. & . & . & . & . . & - & - & - \\
\hline$\beta$-Naphthol. . & • & . & $\cdots$ & . & . . & deutlich & - & - \\
\hline Nitrobenzol. & . . & . & . & . &.+ & - & - & - \\
\hline Tanuin. . & & - & . & . & . . & - & schwach & - \\
\hline Zackerfarbe . & . & . & . & . & . . & deutlich & - & - \\
\hline Hypnon . & & - & . & 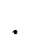 & . & - & - & $\ldots$ \\
\hline Benzaldehyd . & . & . & . & & . & deutlich & deutlich & deutlich \\
\hline Vanillin & & & 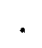 & & & deutlich & deutlich & deutlich \\
\hline Ätherausschüt & tel & ans & aus & $\mathrm{Bi}$ & er. & dentlich & - & - \\
\hline
\end{tabular}

Sulfophthalsäuren geben natürlich die Reaktion, was jedoch nicht von Bedeutung ist, da sie in Nahrungsmitteln nicht vorkommen. Aber auch die Phthalsäuren müssen wir hier mit einrechnen, sie geben zwar keine Sulfoverbindungen, wohl aber Phthaleine, and es ist uns nicht geglückt, durch einfache Reaktionen einen Unterschied zwischen Phthaleinen und Sulfophthaleinen zu finden, sie verbalten sich wegen ihrer nahen Verwandtsehaft fast gleich; in Nahrungsmitteln kommen Phthalsäuren ebenfalls nicht vor.

Ferner geben eine positive Reaktion jene aromatischen Aldehyde, bei denen sich die Aldehydgruppe direkt am Kern befindet, z. B. Benzaldehyd, oder die sich von diesem durch Substitution im Kern ableiten lassen. Befindet sich dagegen die Aldehydgruppe in der Seitenkette, wie beim Zimtaldehyd, $\mathrm{C}_{6} \mathrm{H}_{5} \cdot \mathrm{CH}=\mathrm{CH}$. $\mathrm{CHO}$, so reagieren die Körper nicht. Die aus den Aldehyden entstehenden Farbstoffe sind aber keine Phthaleine, sondern gehören der Gruppe der Triphenylmethanfarbstoffe an, sie sind aber 
ähnlich zusammengesetzt. Diese Aldehyde stören jedoch nicht, da der Ausführung der Reaktion das Abrauchen mit Salzsäure vorausgeht, dem die meisten nicht standhalten. Sie verflüchtigen sich, Benzaldehyd geht in Benzoesäure über, welche die Farbstoffreaktion nicht mehr gibt, usw. Will man aber den gebildeten Farbstoff trotzdem einer näheren Prüfung unterziehen, so ist dies sehr einfach, da die Triphenylmethanfarbstoffe. durch Zusatz von einigen Tropfen Schwefelammonium in alkalischer Lösung sofort zu farblosen Leukoverbindungen reduziert werden, während die Phthaleine und Sulfophthaleine unverändert bleiben.

Ein sehr merkwürdiges Verhalten zeigt aber das Vanillin. Kastle fand, daf Vanillin mit Phenol und konc. Schwefelsäure schon in der Kälte reagiert, während sonst ein Erhitzen auf $180^{\circ} \mathrm{C}$ notwendig ist. Mischt man Vanillin mit Phenol und konc. Schwefelsäure unter guter Kühlung, so färbt sich die Mischung sofort intensiv rot. Wenn man gleich darauf mit Wasser verdünnt, so verschwindet die Farbe wieder, und die Lösung bleibt auch beim Alkalisieren farblos. Läßt man jedoch einige Zeit in der Kälte stehen und verdünnt dann erst mit Wasser, so erhält man eine gelbe Lösung, die beim Zusatz von Alkali blaurot wird. Durch Erwärmen wird die Farbstoffbildung beschleunigt.

Wie nach $\mathrm{Kastle}$, so gibt also auch nach unserer Arbeitsweise das Vanillin

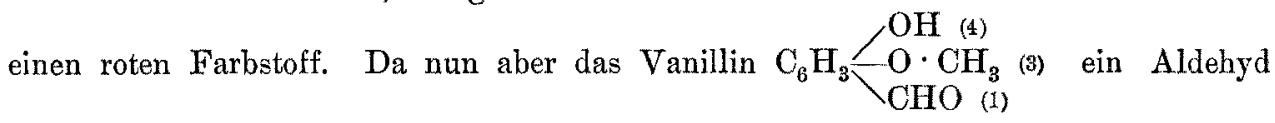
ist, der sich vom Benzaldehyd durch Substitution im Kern ableitet, so müßte der entstehende Farbstoff auch zur Triphenylmethanreihe gehören. Das scheint aber nicht der Fall zu sein, da er durch Schwefelammonium nicht entfärbt wird und sich wie Phenolphthalein und Sulfophenolphthalein aus saurer Lösung mit Äther ausschütteln läßt. Andererseits ist aber die Bildung von Phenolphthalein aus Vanillin nicht sehr wahrscheinlich, da dieses in der o-Stellung zur Aldehydgruppe nicht substituiert ist. Immerhin wären ja Umlagerungen nicht ausgeschlossen.

Das Vanillin wird bei der Herstellung von Konditoreiwaren und Likören verwendet, und da der aus Vanillin sich bildende Farbstoff sich wie ein Phthalein verhält, das Vanillin auch beim Kochen mit Salzsäure sich nicht verflüchtigt oder zersetzt und seinen Aldehydcharakter auch nicht verliert, so ist es allerdings geeignet, die Anwesenheit von Saccharin vorzutäuschen.

Bei unseren Versuchen stießen wir also nur beim Vanillin auf Schwierigkeiten, welches daher vor Ausführung der Reaktion entfernt werden muß. Das gelingt durch Ausziehen des mit Salzsäure abgerauchten Rüekstandes der Äther-Petrolätherauschütte. lung mit Äther und Chloroform zu gleichen Teilen. In diesem Gemenge ist Vanillin leicht löslich, ebenso die meisten übrigen organischen Beimengungen, darunter auch die Paraverbindungen, die im Saccharin vorkommen, nicht aber das saure o-sulfobenzoesaure Ammonium. Dieses bleibt ungelöst zurück.

Versuchen wir, uns über die Eindeutigkeit der Reaktion Klarheit zu verschaffen, so müssen wir die chemischen Vorgänge näher ins Auge fassen, die bei der Farbstoffbildung vor sich gehen. Zunächst steht fest, daß für einen positiven Ausfall der Reaktion nur solche Körper in Betracht kommen können, welche durch Kondensation mit Phenol Phenolphthalein oder Sulfophenolphthalein zu bilden vermögen. Aus der 


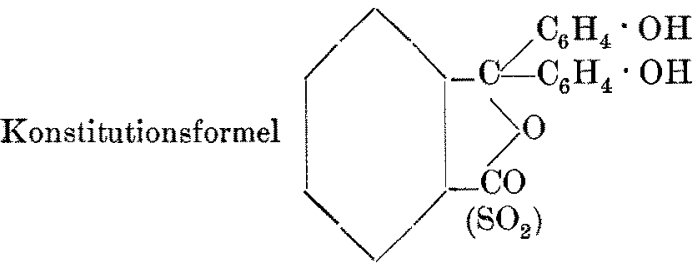

dieser beiden Farbstoffgruppen, welche

einander sehr ähnlich sind, ergibt sich also, daß die Ausgangskörper folgende Merkmale besitzen müssen: Es muß ein Benzolkern vorhanden und an diesem ein Wasserstoffdurch ein Koblenstoffatom substituiert sein, von dem zwei Valenzen so gebunden sind, daß sich je ein Molekül Phenol anlagern kann. Das ist aber nur möglich, wenn diese Valenzen an Sauerstoff oder an Halogen gebunden sind. Die dritte Valenz des Kohlenstoffatoms muß eine OH-Gruppe tragen. Ferner muß sich in Ortbo-Stellung zu diesem Kohlenstoffatom eine saure Gruppe befinden, welche mit der OH-Gruppe des ersten Koblenstoffatoms unter Wasserabspaltung eine Laktonbindung eingehen kann. Das vermag nur die Carboxyl- oder die Sulfogruppe. Anderen Radikalen mit saurem Prinzip, wie der Nitrogruppe oder den Halogenen, kommt diese Eigenschaft nicht zu. Doch ist es nicht nötig, dab die Carboxyl- oder Sulfogruppe als solche vorhanden ist; es können auch Säureamide oder Säurechloride sein.

Es sind nur wenige Körper, welche diesen Forderungen entsprechen, und sie lassen sich alle von zwei Grundsubstanzen ableiten, nämlich von der o-Phthalsäure und von der o-Sulfobenzoesäure. Diese beiden oder ihre Derivate kommen aber in Nahrungs- und Genubmitteln a priori nicht vor, und von Stoffen, welche künstlich hergestellt und in der Nahrungsmittelindustrie verwendet werden, kommt bisher nur das Saccharin in Frage. Die im Saccharin enthaltenen Paraverbindungen können die Reaktion nicht geben, und die die Ermittelung des Sacharins oft erschwerenden Konservierungsmittel, wie Salicylsäure und Benzoesäure, ebenfalls nicht. Wir kommen daher zu dem Schluß, daß der positive Ausfall der Reaktion praktisch eindeutig ist und nur der o.Sulfobenzoesäure, dem süßenden Anteil des Handelssaccharins, zukommt. Auf diese Weise ist also die Gesamtgruppe des Saccharins, mit Ausnahme der Imidgruppe, durch eine einzige leicht ausführbare Reaktion nachgewiesen.

Die Imidgruppe ist auch einfach nachzuweisen, denn sie wird beim Behandeln des Saccharins mit Salzsäure in die Ammoniumgruppe übergeführt und als solche mit Nessler's Reagens erkennbar. Dieser Nachweis empfiehlt sich überhaupt als einfache Vorprobe; ist er negativ, so kann Sacharin nicht vorhanden sein. Ein positiver Ausfall spricht allerdings noch nicht für die Gegenwart von Saccharin, da auch Aldehyde und andere Ammoniumverbindungen die Reaktion geben. Die neben dem Imid vorhandene Amidgruppe der p-Sulfobenzoesäure stört dagegen nicht, da sie beim Behandeln mit Salzsäure unverändert bleibt und auf Nessler's Reagens nicht einwirkt. Bei negativem Ausfall dieser Reaktion ist also jede weitere Prüfung auf Saccharin unnötig.

Die Ermittelung von Saccharin in Nahrungsmitteln gestaltet sich also unter Berücksichtigung dieser Ausführungen einfach folgendermaßen:

Es wird durch Ausschütteln aus saurer Lösung mit Äther-Petroläther das Saccharin gewonnen und das Lösungsmittel in einem Kölbchen verdunstet. Der Rückstand wird mit 10\%-iger Salzsäure einige Minuten gekocht und die Lösung auf dem Wasserbade in einem Schälchen zur Trockne verdampft. Zeigt sich ein Geruch nach 
Vanillin, so wird der Rückstand mehrmals mit $10 \mathrm{ccm}$ einer Mischung von gleichen Teilen Ather-Chloroform kalt ausgezogen, bis der Geruch verschwunden ist. Will man sich aber auf die Geruchsprüfung nicht verlassen, so löst man einen Teil des Ungelösten in Schwefelsäure und setzt etwas Phloroglucin hinzu. Bei Gegenwart von Vanillin entsteht schon in der Kälte eine intensive Rotfärbung.

Bei Abwesenheit von Vanillin prüft man zunächst einen Teil des Salzsäurerückstandes mit Nessler's Reagens. Bei negativem Ausfall der Reaktion kann kein Saccharin vorhanden sein, und die weitere Untersuchung erübrigt sich. Bei positivem Ausfall wird der Salzsäurerückstand in etwas Phenol gelöst und in einem Porzellantiegel auf Phosphorpentoxyd getropft. Die Bildung eines roten Farbstoffes, der sich in Wasser mit gelber Farbe löst und auf Zusatz von Alkali blaurot wird, beweist die Anwesenheit von Saccharin. Zur Sicherheit können noch wenige Tropfen Schwefelammonium zugesetzt werden, die Farbe darf nicht sofort verschwinden.

Zur quantitativen Bestimmung des Saccharins ist zu bemerken, daß sich dazu zwei Methoden eignen, die Ausmittelung der Sulfosäure und des Imidstickstoffs. Bei der ersten ist zu beachten, daß nicht nur die Schwefelsäure, sondern auch Schwefel bestimmt wird, auch wenn man nicht mit Soda und Salpeter, sondern mit Soda und Kaliumchlorat aufschließt, und daß auch die Parasulfoverbindungen mitbestimmt werden. Man erhält also, falls nicht chemisch reines Saccharin vorliegt, viel zu hohe Werte. Es ist deshalb notwendig, nicht den Rückstand des ätherischen Auszuges, wie es bisher üblich war, zur Bestimmung zu benutzen, sondern ihn vorher mit Salzsäure zu hydrolysieren, mit Äther-Chloroform von Paraverbindungen zu befreien und dann erst im Rückstand die Sulfogruppe zu ermitteln.

Die Entfernung der Paraverbindungen ist vor der Ausmittelung von Saccharin unerläßlich, da sie nicht unter das Süßstoffgesetz fallen.

\section{Referate.}

\section{Ernährungslehre.}

Charles G. L. Wolf: Eiweibstoffweehsel nach Hunger und Aufnahme großer Mengen körpereigenen und körperfremden EiweiBes. (Biochem. Zeitschr, 1914, 63, 58-73.) - An Hunde wurde artfremdes (Rinderherz) und arteigenes (Hundefleisch) Protein verfüttert und die Ausnutzung beider ermittelt. Die Versuche stützen die Auffassung, daß arteigenes Protein für die Ernährung vollwertiger ist als artverschiedenes, indem die Retention von Stickstoff, Schwefel, Phosphor, Calcium und Chlor nach Verfütterung von körpereigenem Protein erheblich größer war. W. Sutthoff.

E. Grafe: Beiträge zur Kenntnis der Artder Stickstoffretentionen bei Füterung von Ammoniaksalzen und Harnstoff. (Zeitschr. physiol. Chem. 1913, 88, 389-424.) - In Verfolg früherer Arbeiten berichtet Verf. über Fütterungsversuche an Schweinen mit Ammoniaksalzen und Harnstoff, die den Zweck hatten, die Art der Stiokstoffretentionen festzustellen. Die Hauptergebnisse sind kurz folgende: Eine allmähliche Gewichtszunahme $\nabla 0 n 2800 \mathrm{~g}$ in 20 Tagen bei einem Tiere, das nur einen. Teil der Abnutzungsquote in Form von Eiweiß erhielt und ohne Harnstoff- und Ammoniakzulagen mindestens $0,3-0,5 \mathrm{~g}$ an Stickstoff verloren hätte, spricht dafür, daß die dauernd retinierten Mengen von Stickstoff in eiweißartiger,Form 